\title{
Percepções de aspectos psicossociais no cuidado em saúde de adolescente com obesidade grave
}

\section{Perceptions of psychosocial aspects in health care of adolescents with severe obesity \\ Percepciones de los aspectos psicosociales en el cuidado en salud de adolescente con obesidad grave}

\author{
Adriana Lobo Müller ${ }^{1}$ \\ Universidade Regional de Blumenau (FURB) \\ Cláudia Regina Lima Duarte da Silva \\ Universidade Regional de Blumenau (FURB) \\ Deisi Maria Vargas \\ Universidade Regional de Blumenau (FURB)
}

\begin{abstract}
Resumo
O objetivo deste estudo foi desvelar percepções de aspectos psicossociais no cuidado de adolescentes com obesidade grave na atenção básica. Trata-se de um estudo qualitativo - descritivo que utilizou a entrevista com roteiro semiestruturado para coleta de dados. Os dados foram processados e analisados através de análise temática de conteúdo. Foram entrevistados seis profissionais de saúde, cinco adolescentes com obesidade grave e seis familiares de adolescentes com obesidade grave. Foram identificadas três categorias temáticas: concepção de obesidade, concepção de cuidado e obesidade na vida. Os dados encontrados evidenciaram que a concepção de obesidade dos profissionais de saúde, adolescentes e familiares é multifatorial, incluindo os fatores genéticos e orgânicos, o modo de vida contemporâneo e os aspectos psicológicos. A concepção de cuidado envolveu dieta, atividade física e cirurgia bariátrica. Nessa perspectiva foram realizadas reflexões acerca das políticas públicas de nutrição, saúde mental e saúde do adolescente permeadas pela proposta emergente da integralidade em saúde.
\end{abstract}

Palavras-chave: adolescência, obesidade, aspectos psicossociais, percepção, atenção primária em saúde

\section{Abstract}

The aim of this study was to revel perceptions of psychosocial aspects in the care of adolescents with severe obesity at primary health care. It is a qualitative and descriptive study that used semistructured interview for data collection. Thematic content analysis was used to process and analyse text data. Six health professionals, five adolescents with severe obesity and six relatives of adolescents with severe obesity were interviewed. Three categories were identified: obesity conception, health care conception and obesity in life. Our findings show that the conception of obesity by health professionals, adolescents and family was multifactorial including genetic and organic factors, the contemporary way of life and psychological aspects. The health care conception was characterized by diet, physical activity and bariatric surgery. From this perspective, the authors did some reflections on the Brazilian public policies on nutrition, mental health and adolescent health based on the emerging concept of comprehensive health care.

Keywords: adolescence, obesity, aspects psychosocial, perception, primary health care

\section{Resumem}

El objetivo de este estudio ha sido desvelar percepciones de los aspectos psicosociales en el cuidado de adolescentes con obesidad grave en la atención primaria. Es un estudio cualitativo- descriptivo que utilizó la entrevista semi-estruturada. Los datos fueron procesados y analizados a través del análisis temático de contenido. Se entrevistó a seis profesionales de la salud, cinco adolescentes con obesidad grave y seis familiares. Se identificaron tres categorías temáticas: concepción de obesidad, concepción de cuidado y obesidad en la vida. Los datos encontrados evidenciaron que la concepción de obesidad de los entrevistados es multifactorial, incluyendo los factores genéticos y orgánicos, el modo de vida

\footnotetext{
${ }^{1}$ Endereço de contato: Rua José Deeke, 1596, apto. 106, Escola Agrícola Blumenau, SC. CEP 89031-401. E-mail: adrilmuller@hotmail.com
} 
contemporáneo y los aspectos psicológicos. La concepción de cuidado involucró la dieta, la actividad física y la cirugía bariátrica. En esa perspectiva se realizaron reflexiones acerca de las políticas públicas de nutrición, salud mental y salud del adolescente permeadas por la propuesta emergente de la integralidad en salud.

Palabras clave: adolescência, obesidade, aspectos psicosociales, percepción, atención primária de salud

\section{Introdução}

A obesidade é um agravo multifatorial caracterizado pelo acúmulo excessivo de gordura, resultante do balanço energético positivo (Brasil, 2006a). A multifatorialidade da obesidade está relacionada à interação de fatores como genética, idade, raça, nível de atividade física, perfil alimentar e consumo de álcool (Brasil, 2006a; Brasil, 2014a). Além disso, fatores culturais, sociais, econômicos e psicológicos também influenciam no fenômeno (Brasil, 2006a; Brasil, 2014a; Schoen-Ferreira, Aznar-Farias, \& Silvares, 2010; França, Sahade, Nunes, \& Adan, 2013; Kaufman, 2012; Loli, 2000). No Brasil, dados indicam que, entre os anos de 2008 e 2009, a prevalência de excesso de peso em crianças em idade escolar e adolescentes chegou a 34,8\% para meninos e 32,0 \% em meninas. Comparando os períodos de 1974- 1975 e 2008- 2009, "a prevalência de excesso de peso aumentou em seis vezes no sexo masculino (de 3,7\% para $21,7 \%$ ) e em quase três vezes no sexo feminino (de 7,6\% para 19,4\%)" (Brasil, 2014a, p. 10).

Do ponto de vista psicossocial, comer mais do que o necessário, de forma a exceder a saciedade, é uma forma de utilizar a alimentação para preencher algo que não está na instância física, mas sim, psíquica. O comer, nessa concepção, é uma tentativa de aliviar tensões, ansiedades e angústias (Carneiro, 2005; Alves \& Laguardia, 2012; Kaufman, 2012). Nessa perspectiva, a obesidade é uma "expressão através do corpo de algo que realmente não pode ser expresso pela via da fantasia nem pela via do pensamento e da linguagem" (Loli, 2000, p. 78), assim, os alimentos podem ser usados como recurso para o sujeito aliviar frustações e ansiedades, mascarando um conflito psíquico (Conejo, 2009). Os significados psíquicos da obesidade variam para cada sujeito, sendo recorrentemente utilizada como mecanismo de defesa, camuflagem da agressividade, autocastigo e manejo de esquiva, e proximidade de aspectos da sexualidade (Burd, 2010). Assim, a obesidade é uma importante fonte de informação sobre a história de vida dos sujeitos obesos e contam como estes enfrentam as dificuldades do mundo interno e externo (Conejo, 2009).

A adolescência é considerada pelas Diretrizes Nacionais para a Atenção Integral à Saúde de Adolescentes e Jovens como um processo complexo marcado por três dimensões interdependentes: a primeira macrossocial, que considera as desigualdades sociais, isto é, gênero, classe e etnia; a segunda engloba os dispositivos institucionais como escola e trabalho; e por fim, a terceira, chamada biográfica, que considera as singularidades da história pessoal de cada adolescente (Brasil, 2010, p. 46).

No contexto da obesidade durante a adolescência, a família e os profissionais de saúde, apresentam-se como atores fundamentais tanto para a manutenção da obesidade, quanto para seu cuidado. Ao compreender a adolescência como uma fase da vida que não se resume a transformações fisiológicas e universais da puberdade, desperta-se para um olhar mais reflexivo acerca das transformações psicossociais desse período. Tais transformações imprimem o entendimento de que não há uma adolescência, e sim, várias adolescências, 
pois, consideram as características do contexto sociocultural e pessoal de cada sujeito, o que influencia nas diversas possibilidades de se viver essa etapa da vida (Schoen-Ferreira, AznarFarias, \& Silvares, 2010).

Diante do exposto, o objetivo deste estudo foi desvelar as percepções acerca dos aspectos psicossociais no cuidado de adolescentes com obesidade grave na perspectiva do adolescente e de seus cuidadores (responsáveis e profissionais).

\section{Procedimentos Metodológicos}

\section{Desenho}

O estudo trata-se de pesquisa qualitativa, descritiva e exploratória situada na área da saúde coletiva, na linha da Promoção à Saúde e Integralidade do Cuidado de um Mestrado Profissional em Saúde Coletiva. Foi realizada junto a um projeto institucional de extensão da universidade que comporta o mestrado.

\section{Participantes}

Os sujeitos da pesquisa foram seis profissionais de saúde (quatro enfermeiros, um dentista, um profissional da educação física), cinco adolescentes com diagnóstico de obesidade grave e seis familiares (Tabela 1). Os critérios de inclusão dos profissionais de saúde foram: ser profissional de nível superior das unidades Estratégia de Saúde da Família (ESF) vinculadas projeto de extensão, ou profissional de nível superior da equipe técnica do projeto. Os critérios de inclusão dos adolescentes foram: ser cadastrado em ESF vinculada ao projeto de extensão e ter diagnóstico de obesidade grave conforme critérios estabelecidos pela Organização Mundial da Saúde (Brasil, 2014c). Os critérios de inclusão dos familiares foram: ter vínculo familiar com adolescente que atendia aos critérios de inclusão dessa pesquisa. Os sujeitos da pesquisa foram selecionados dentro do universo do cadastro de adolescentes com excesso de peso do projeto de extensão que continha os dados de todos os adolescentes com excesso de peso adscritos à área de abrangência de quatro equipes de Estratégia de Saúde da Família. Foram identificados doze núcleos familiares que atendiam aos critérios de inclusão, sendo que cinco se negaram a participar alegando que não tinham interesse.

\section{Procedimentos de Coleta de Dados}

A coleta de dados foi realizada por meio de entrevistas individuais com roteiro semiestruturado. Foi elaborado um roteiro específico para cada ator entrevistado (adolescente, familiar e profissional). Após autorização por escrito de cada participante e seu responsável, as entrevistas foram gravadas em áudio e posteriormente transcritas. As entrevistas foram realizadas pela pesquisadora responsável após agendamento por contato telefônico. A coleta de dados aconteceu no período de 10 de setembro a 29 de outubro de 2015.

\section{Análise de Dados}

O tratamento dos dados foi centrado na análise temática de conteúdo proposta por Bardin (2010), que tem por finalidade interpretar o não dito, o oculto nas mensagens e unidades 
linguísticas, isto é, toma os significados como material dominante. Após a análise, foram estabelecidas três categorias temáticas, sendo elas: concepção de obesidade, concepção de cuidado e obesidade na vida. Por questões éticas e de sigilo, os sujeitos foram identificados pelos seguintes códigos: " $P$ " para profissionais de saúde, " $A$ " para adolescentes e " $F$ " para familiares. Cada sujeito recebeu um código em letra ( $P, A$ ou F) e um numeral para diferenciá-los. Para identificar os núcleos familiares, adolescentes e familiares receberam o mesmo numeral.

\section{Aspectos Éticos}

O projeto foi aprovado pelo comitê de ética de pesquisa em seres humanos da FIURB através do parecer n. 1.193.544 em 20 de agosto de 2015.

Tabela 1

Caracterização dos núcleos familiares

\begin{tabular}{|c|c|c|c|}
\hline $\begin{array}{l}\text { Núcleo } \\
\text { familiar }\end{array}$ & Entrevistados & $\begin{array}{c}\text { Idade do } \\
\text { adolescente }\end{array}$ & $\begin{array}{c}\text { Sexo do } \\
\text { Adolescente }\end{array}$ \\
\hline 1 & $\begin{array}{c}\text { A1 } \\
\text { F1 (mãe) }\end{array}$ & 16 & Feminino \\
\hline 2 & $\begin{array}{c}\text { A2 } \\
\text { F2 (mãe) }\end{array}$ & 14 & Masculino \\
\hline 3 & $\begin{array}{c}\text { A3 } \\
\text { F3 (mãe), } \\
\text { F4 (padrasto) }\end{array}$ & 19 & Masculino \\
\hline 4 & A4 & 18 & Masculino \\
\hline 5 & $\begin{array}{c}\text { A5 } \\
\text { F5 (mãe) }\end{array}$ & 13 & Feminino \\
\hline 6 & A6 & 19 & Feminino \\
\hline 7 & F6 & 15 & Feminino \\
\hline
\end{tabular}

\section{Resultados}

Os resultados da pesquisa foram elencados em três categorias temáticas, sendo elas: concepção de obesidade, concepção de cuidado e obesidade na vida.

\section{Concepção de Obesidade}

A categoria concepção de obesidade permeou a fala de todos os sujeitos entrevistados e, pela diversidade dos discursos, foi subdividida em: "aspectos genéticos e orgânicos"; "cultura, sociedade e contemporaneidade"; e "aspectos psicológicos e relações familiares".

A subcategoria "fatores genéticos e orgânicos" apresentou concepção de obesidade permeada por determinantes biológicos e associada a outras condições clínicas por todos os atores. Tal concepção pode ser considerada herança da concepção biomédica que produz um discurso normatizador sobre os modos de vida, compreendendo de forma reducionista e objetificadora os processos de adoecimento e normalidade (Nunes \& Pelizzoli, 2011). 
Porque assim, a gente sabe que a obesidade tem a parte genética, a parte doença.... ela já tem aquela adiposidade toda para desenvolver, né? Aquelas células, os adipócitos que não são poucos. (P3)

Só que eu tenho tireoide também. (A3)

A partir do colégio foi que a gente começou a descobrir que ele era ansioso, que ele tinha déficit de atenção. . . Foi o que ajudou a desencadear a obesidade dele, porque aí ele não teve mais controle, ele não dormia à noite. Passava noite inteirinha acordado, comendo a noite toda. (F2)

Na subcategoria "cultura, sociedade e contemporaneidade", alguns fatores foram apresentados como desencadeadores e mantenedores da obesidade pelos profissionais. Apresentaram discurso crítico e reflexivo acerca do modo de vida contemporâneo ao compará-lo com o modo de vida de anos atrás. Compreenderam que essas mudanças estimulam o sedentarismo e, por conseguinte, a obesidade.

A gente está observando um aumento significativo da obesidade, principalmente eu acho que pela alimentação, o estilo de vida assim, não come direito e não se exercita o suficiente. (P3)

Hoje em dia é tudo mais fácil. As pessoas têm ônibus na porta de casa, elas não precisam fazer muito esforço físico para chegar na escola. (P6)

Não praticam mais atividade nenhuma, é internet, é vídeo game e não fazem atividade. (P5)

A sobrecarga de compromissos marcada pela entrada da mulher no mercado de trabalho foi percebida pelos adolescentes e profissionais como fator associado à obesidade.

A mãe trabalha em um horário, o pai noutro ..., não estão mais juntos, não tem mais aquela refeição, aquele prazer em estar em família. (P3)

Durante a semana, como minha mãe não tinha tempo de cozinhar, o café era sempre no terminal [de ônibus], era sempre aquele pastel. (A6)

Essa percepção da associação da obesidade com as mudanças do papel social da mulher corrobora com dados encontrados por Dornelles, Anton, e Pizzinato (2014), que identificaram a transição das responsabilidades femininas que, progressivamente, dividem-se entre o âmbito dos cuidados familiares e do mercado de trabalho. No entanto essa transição não foi acompanhada pela responsabilidade compartilhada com os homens da família, o que pode ser atribuído, segundo os autores, ao tradicional estereótipo de gênero da nossa sociedade. Ainda nessa temática, Simão e Mioto (2016) apresentam dados de países da América Latina sobre a taxa de ocupação das mulheres no mercado de trabalho remunerado, apontando que, no Brasil, a taxa atinge 50,8\%, apresentando o país dados mais significativos quando comparados ao Uruguai $(42,69 \%)$ e Argentina (43,5\%). Confirma-se assim "a luta das muIheres por autonomia e ao desvencilhamento do papel de cuidadoras da família" (Simão, \& Mioto, 2016, p. 158).

Na subcategoria "aspectos psicológicos e relações familiares", os discursos apresentaram a compreensão de que o alimento é utilizado como recurso para aliviar angústias e frusta- 
ções. Essas percepções corroboram com a literatura científica ao afirmar que os sentimentos negativos relacionados à obesidade, somados à baixa autoestima, podem gerar crises que resultam no movimento: "comer - angustiar - se - comer" (Rodrigues, \& Boog, 2006, p. 926), que proporciona a manutenção da obesidade. Como o alimento não é capaz de nutrir necessidades afetivas, o comportamento alimentar passa a ser uma engrenagem do ciclo vicioso, no qual, de um lado, há o ganho progressivo de peso e, do outro, há um vazio cada vez maior (Conejo, 2009, p. 62).

Minha percepção é que ele realmente ganhou peso por um sofrimento emocional não visto. (P4)

Eu comia muito por tristeza. Se acontecia alguma coisa que me frustrava, descontava na comida. (A6)

As configurações familiares também são compreendidas como fatores que favoreceram a obesidade. A influência dos avós na educação foi, claramente, evidenciada.

Então ela mora com a avó, também é um fator que eu acho que contribui. A avó quer agradar, diz muito "sim" pra tudo. E eu vejo, a falta do pai e da mãe, então ela mora com a avó e a avó não toma decisões, ela deixa, vai levando né? (P6)

Ainda nas relações familiares, a separação dos pais também consta como causa para o desencadeamento da obesidade, fato que corrobora com Burd (2010, p. 306) "a obesidade pode compensar um sentimento de abandono, quando o indivíduo perde o objeto amoroso....

Ela teve um distúrbio, acho eu, porque eu me separei do pai dela, e aí ela começou a comer, comer e acabou que engordou bastante. (F5)

Diferentemente dos profissionais que apresentaram discursos generalizados sobre a obesidade na adolescência, os familiares conseguiram identificar um evento marcador específico para seu início, como separação dos pais, mudanças de cidade e dificuldade de adaptação ao ingressar na escola.

A partir do colégio foi que a gente começou a descobrir que ele era ansioso, que ele tinha déficit de atenção. . . . Foi o que ajudou a desencadear a obesidade dele, porque aí ele não teve mais controle, ele não dormia à noite. Passava noite inteirinha acordado, comendo à noite toda. (F2)

\section{Concepção de Cuidado}

Apesar da heterogeneidade nos discursos acerca da concepção de obesidade, as propostas de cuidado restringiram-se a recursos e tecnologias direcionadas a orientações nutricionais, combate ao sedentarismo e a intervenções cirúrgicas. As falas de todos os grupos se localizaram na dimensão corporal, desconsiderando outras possibilidades de cuidado, como grupos terapêuticos ou de educação em saúde.

Um estilo de vida mais ativo, mais motivante, poderia mexer em outros fatores do estilo de vida como a alimentação. (P2) 
A gente começou a ficar no pé dela, pedir os exames, orientar a fazer atividade física. (P5)

Chamei ela e disse assim: vamos te encaminhar para cirurgia bariátrica? (P1)

Outro aspecto bastante claro no discurso dos profissionais foi a dificuldade de estabelecer vínculo com os usuários, o que facilitaria a efetivação de uma maior sensibilidade, tanto para o cuidado, quanto para o autocuidado.

E a gente ficou assim, um pouco decepcionado porque o que era problema para nós, não era problema pra ela . . confesso que a gente não consegue sensibilizar a pessoa. . . . (P6)

Eu fico pensando assim: o que é que realmente a gente não atinge nas pessoas? Como nós vamos trabalhar esse psicológico para a pessoa se convencer, realmente, que está perdendo a saúde? (P3)

Diversos autores (Grejanin et al., 2007; França et al., 2013; Rodrigues \& Boog, 2006), afirmam que as dificuldades de abordagem dos sujeitos obesos pelos profissionais de saúde estão permeadas pela formação acadêmica caracterizada pela forte influência da concepção biomédica. Dessa forma, sua prática profissional baseia-se em ações de cuidado e atenção restritas a orientações pedagógicas voltadas somente à dimensão corporal. No caso da obesidade: mudanças alimentares e práticas de atividades físicas. A percepção do sujeito integral não existe. Prevalece a compreensão do julgamento moral de que a pessoa com obesidade não emagrece por falta de força de vontade, e não pela compreensão de que a negação da obesidade pode ter significados psíquicos. A presença dessas atitudes estereotipadas e negativas contribui para que o paciente obeso evite procurar ajuda (Segal, Cardeal, \& Cordas, 2002). Tais limitações são percebidas pelos usuários dos serviços, os quais indicam dificuldade de acesso e falta de vínculo com a equipe de saúde.

Aqui a gente tem um acesso muito ruim. Tu quer uma consulta médica, tu tem que penar pra conseguir. A gente tem uma enfermeira chefe, aqui no postinho, que não é fácil. ... A gente tem a sensação que está sempre devendo alguma coisa para ela. . . Eu só venho aqui em extrema necessidade, senão eu não venho. (F5)

Agora que nem a profissional da saúde que eu chego lá, e ela joga na minha cara que eu engordei aquilo tudo, pra mim foi como se ela tivesse contra mim. . . . Hoje em dia, eu não gosto daquele posto e não vou. (A6)

No que se refere ao cuidado oferecido pelos familiares, nota-se que suas práticas também são direcionadas para a alimentação e atividade física.

Tento que ela tenha hábitos saudáveis. Influencio ela que ela vá a pé e volte da escola. ... Faço ela comer bastante verdura, legumes. (F1)

Antes eu tinha o hábito de ir fazer compra e comprar vários pacotes de bolachinha recheada, chocolate. Ai eu notei que ela engordou muito e comecei a cortar. Eu limitei isso. (F5)

Algumas famílias consideram que a responsabilidade pelas mudanças nos hábitos alimentares é exclusiva dos adolescentes, mantendo, assim, oferta de alimentos que promovem a 
manutenção da obesidade. Apresentam, também, dificuldade de iniciar e manter um diálogo referente às dificuldades com a alimentação e, muitas vezes, demonstram uma postura autoritária e crítica, o que reforça o consumo inadequado de alimentos (França et al., 2013; Rodrigues \& Boog 2006. Corroborando com a literatura, alguns familiares pesquisados, acreditavam que a responsabilidade maior em relação aos cuidados com a obesidade é do próprio adolescente.

Eu acredito que ela já tem idade suficiente para escolher se ela quer fazer ou não, eu só incentivo, mas não aquela coisa, impondo que ela tem que fazer, porque tá gorda. (F1)

Eu digo pra ele: come pouco. E ele for comer pouco, beleza. Se ele come bastante, eu só digo: vai te prejudicar, meu filho. Aí eu deixo ele, pra ele mesmo, por ele mesmo. (F2)

No mesmo direcionamento de profissionais de saúde e familiares, o autocuidado dos adolescentes também se restringiu a mudanças alimentares e práticas de atividade física e à tentativa de seguir regras padronizadas, mesmo que isso resultasse em sofrimento e angústias.

Agora eu faço academia. Na verdade, eu nem gosto de fazer academia, mas ele lo médico) fala que é bom para fortalecer. Eu sempre fui muito comilona, pra mim foi difícil mudar os hábitos, mudar tudo, tanto que no primeiro ano que eu fiz a dieta . . . pra mim foi um sacrifício fazer aquilo. Eu fiquei triste e até hoje eu fico. (A6)

Tem vezes que eu fico um tempo sem comer (guloseimas) . . comida assim não tanta, é mais besteira mesmo. E fora do horário e tal. Quando eu estava estudando à tarde eu tomava café tarde e aí almoçava quase ao mesmo tempo, dai não tinha aquele intervalo de 3 horas que falam. (A1)

\section{Obesidade na Vida}

Os efeitos da obesidade na vida ultrapassam as complicações clínicas. Assim, essa categoria divide-se em duas subcategorias, sendo elas: "preconceito" e "acessibilidade e consumo", que surgiram nas falas dos adolescentes e profissionais. Mattos (2012, p. 25), entende que "ao corpo se aplicam crenças, sentimentos, desejos, sonhos, realizações e sentidos que estão na base da vida social", assim o corpo é um sistema simbólico que transmite mensagens mesmo que não tenhamos consciência disso. Nessa perspectiva, o autor compreende que o corpo gordo, diante da atual cultura do corpo "sarado", representa sentidos e valores depreciativos e desvalorizados, como sujo, perdedor, fracassado, preguiçoso, e completa: o horror à gordura é imperativo numa sociedade cercada por espelhos (Mattos, 2012). Na subcategoria "preconceito", tal perspectiva foi encontrada no discurso dos sujeitos entrevistados:

Dava impressão que vinha até suja. . . Não é que ela vinha suja, fedendo, mas era um desleixo tão grande que dava essa impressão. (P1)

Eu acho que a pessoa tem a visão de que obeso é lento, e talvez isso seja uma verdade mesmo. (P6)

Eu sempre escutava as pessoas comentando. Por exemplo, sobre usar um biquíni na praia, você tem que usar maiô! Você não pode usar biquíni. (A6) 
As dificuldades relacionadas com a obesidade também se apresentam quando o adolescente precisa andar de ônibus ou comprar roupas. Na subcategoria "acessibilidade e consumo", verificaram-se os seguintes relatos:

Eu lembro que, para sentar em banco de ônibus, eu sempre sentava sozinha, porque eu tinha muita vergonha de sentar em dois. (A6)

Ela já não passava na catraca do ônibus ... como é que a pessoa vai andar de ônibus se a pessoa não passa na catraca? ... tem que entrar por trás, junto com os idosos. (P6)

Eles fazem muito roupa pequena, dai nunca tem a roupa grande que eu quero. (A5)

A falta de acessibilidade para obesos, geralmente, está relacionada às questões dimensionais, isso é, espaços reduzidos em poltronas, catracas, banheiros e vestuários (Menin, Paschoarelli, \& Silva, 2011).

Eu me lembro que eu ia comprar uma calça jeans e eu não encontrava, aí isso me frustrava, eu chegava em casa e chorava. (A6)

\section{Discussão}

O objeto desta pesquisa foi a obesidade na adolescência, mais especificamente o cuidado aos aspectos psicossociais envolvidos nesse fenômeno. De acordo com o tratamento dos dados, foram encontrados discursos, tanto de profissionais de saúde quanto de adolescentes e familiares, que apresentaram concepções de obesidade na adolescência permeadas pela multifatorialidade. Foram encontradas concepções que atribuem a obesidade a fatores genéticos, ao modo de vida contemporâneo, e também opiniões indicadoras de que aspectos psicológicos influenciam no modo de comer de adolescentes, como comer por ansiedade ou tristeza, e não por fome.

Fatores como as novas configurações familiares, a influência dos avós na educação, a mudança do papel social da mulher marcada pela sua entrada no mercado de trabalho e eventos marcadores, como separação dos pais, mudança de cidade e entrada na escola, também foram citados como desencadeadores ou mantenedores da obesidade. Nesse sentido, a pesquisa mostrou a importância e influência dos aspectos psicossociais no fenômeno da obesidade na adolescência. Os achados também indicaram que a concepção e oferta de cuidado estão, prioritariamente, direcionados para a mudança nas configurações corporais e controle de peso, índice de massa corporal (IMC) e outros marcadores anatomofisiológicos de doença.

O aumento epidemiológico da obesidade exige das políticas públicas ações efetivas no combate à obesidade comprometidas com as diretrizes do SUS, como ações de promoção, prevenção e tratamento (Brasil, 2006a; Brasil,2014a). São exemplos de políticas públicas, consoantes com o cenário epidemiológico do país na atualidade, a PNAN, que visa melhorar as condições de alimentação, nutrição e saúde da população brasileira (Brasil, 2012), e o Guia Alimentar para a População Brasileira, que apresenta recomendações para uma alimentação saudável e serve de instrumento para educação alimentar e nutricional no SUS (Brasil, 2014b).

Em contrapartida às políticas públicas de nutrição, que, apesar de considerarem a alimentação como expressão das relações sociais, valores e história do indivíduo, são claramente 
direcionadas ao controle epidemiológico da obesidade através de orientações pautadas em marcadores anatomofisiológicos (Brasil, 2012), há três documentos do Ministério da Saúde (MS) norteadores para a efetivação do cuidado integral e da observância dos aspectos psicossociais da obesidade na adolescência, sendo eles as Diretrizes Nacionais para a Atenção Integral à Saúde de Adolescentes e Jovens na Promoção, Proteção e Recuperação da Saúde, Cadernos de Atenção Básica - Estratégias Para o Cuidado da Pessoa com Doença Crônica Obesidade (Brasil, 2014c) e o Caderno de Atenção Básica - Saúde Mental (Brasil, 2013).

Nas Diretrizes Nacionais para a Atenção Integral à Saúde de Adolescentes e Jovens na Promoção, Proteção e Recuperação da Saúde, são descritas particularidades da população adolescente que a diferenciam de crianças e adultos e, a partir dessa perspectiva, propõem ações de promoção de saúde que convoquem os adolescentes para a construção participativa de ambientes saudáveis, ampliando as redes de compromisso e de corresponsabilização. Essa convocação favorece o exercício da cidadania através do planejamento e avaliação das ações de saúde (Brasil, 2010).

Nos Cadernos de Atenção Básica - Estratégias Para o Cuidado da Pessoa com Doença Crônica Obesidade, há orientações de abordagens para o manejo do sobrepeso e da obesidade em adolescentes que contemplam a inserção no mundo do trabalho, a influência dos amigos e da mídia em suas escolhas alimentares e questões relacionadas à sexualidade. As orientações prezam pela disponibilidade, flexibilidade e sensibilidade para acolher, escutar e compreender as particularidades dessa etapa da vida. Apesar de claras orientações para estimular a adoção de uma alimentação saudável e prática de exercícios físicos, esse documento apresenta uma perspectiva de cuidado que estimula o autoconhecimento, a promoção da autonomia através da construção de maiores capacidades de análise e de corresponsabilização pelo cuidado consigo e com os outros, bem como uma atitude protagonista diante da vida (Brasil, 2014c).

Por fim, o Caderno de Atenção Básica - Saúde Mental estimula a desmistificação acerca do sofrimento psíquico, indicando que os cuidados em saúde mental acontecem no cotidiano da atenção básica, assim, propõe intervenções que valorizem as queixas emocionais dos usuários como legítimas, através de um olhar sobre o sujeito que considere suas múltiplas dimensões, desejos, valores e escolhas como fatores passíveis de promover modificações nas condições e hábitos para a produção de vida e saúde. Muitas vezes, o usuário não percebe a relação de seus conflitos e seus sofrimentos com seu modo de ser na vida e "ter o profissional de Saúde da Atenção Básica como um interlocutor pode ser uma via para lidar com esses sofrimentos cotidianos, muitas vezes responsáveis por somatizações ou complicações clínicas" (Brasil, 2013, p. 24).

A contribuição mais relevante desse documento é o olhar sobre adolescência, pois considera a singularidade dos sujeitos trazendo assim, para o foco do cuidado em saúde, a perspectiva de que cada um "tem sua própria história, seu jeito de ser, seu modo de aprender com experiências por quais passa, suas questões subjetivas, familiares e sociais, suas dificuldades, e suas tentativas de solução" (Brasil, 2013, p. 105).

Esses três documentos constituem para o cuidado a adolescentes obesos uma tríade que engloba a integralidade desses sujeitos, sendo composta por: adolescente enquanto cidadão corresponsável pela construção de ambientes saudáveis; abordagem ao adolescente 
considerando as especificidades dessa etapa da vida; estímulo à autonomia e protagonismo do adolescente enquanto sujeito singular e dono de uma história única.

Compreende-se, a partir deste estudo, que existe um hiato entre a normativa teórica e científica dada pelas políticas públicas que colocam a integralidade em saúde como diretriz para as ações em saúde, e a oferta de cuidado integral a adolescentes obesos. A integralidade significa a oferta de cuidado nos campos da promoção, prevenção, cura, reabilitação e cuidados paliativos, através do reconhecimento adequado dos problemas biológicos, psicológicos e sociais (Mendes, 2012).

Diante dessa perspectiva, faz-se necessário repensar no âmbito da oferta de cuidados da atenção básica a partir de qual horizonte normativo está permeada a atuação profissional. Considerando que esses horizontes normativos são construídos socialmente, Ayres (2004), ao discutir o cuidado e as práticas de saúde, indica que a interação terapêutica é orientada pelo horizonte normativo, isso é, se o horizonte normativo é a morfuncionalidade e seus riscos, a oferta terapêutica será direcionada para encontrar subsídios com objetivos que resultem no êxito técnico, no caso da obesidade, seriam a redução de peso e IMC, por exemplo. Por outro lado, quando o horizonte normativo é ampliado para uma dimensão existencial em busca de significados e sentidos para a saúde e para o projeto de vida, a oferta terapêutica objetivará o sucesso prático, ou seja, o alcance da felicidade num sentido existencial.

No intuito de apresentar respostas que envolvam mudanças no perfil epidemiológico da obesidade, o cuidado aos aspectos psicossociais e a compreensão do homem enquanto ser integral são aspectos preteridos por mudanças em estatísticas epidemiológicas. Considerar a subjetividade dos adolescentes, auxiliá-los a compreender o significado da comida e da obesidade em sua vida, suas relações afetivas e modos de viver, implica ultrapassar a atenção centrada na doença para a atenção centrada na pessoa. Essa é a necessária "habilidade de fazer conexões entre a generalidade da ciência biomédica e a experiência singular, objetiva ou subjetiva, consciente ou inconsciente, de uma pessoa portadora de doença ou de enfermidade" (Mendes, 2012, p. 244).

Na complexidade da obesidade na adolescência, o modelo de atenção centrado na pessoa prevê o reconhecimento da obesidade como possibilidade de denúncia de sofrimento psíquico e, por isso, demanda a humanização do cuidado, ou seja, “. . . Cuidado como designação de uma atenção à saúde imediatamente interessada no sentido existencial da experiência de adoecimento, físico ou mental. .." (Ayres, 2004, p. 22), superando assim, o cuidado operativo de aplicação das tecnociências (Ayres, 2004).

Por essa perspectiva, ser ou manter-se obeso tem sentidos diferenciados para cada adolescente e cada contexto familiar. Assim, empenhar-se em emagrecer, lançar-se em dietas, assumir-se enquanto praticante de atividade física pode ter efeitos distintos na saúde mental de cada sujeito. Igualmente, ser ou manter-se obeso é uma condição psíquica que se desdobra em diversas possibilidades, como representar um mecanismo de defesa, um recurso para sentir-se pertencente e fiel ao núcleo familiar ou, ainda, uma denúncia sobre o modo que o adolescente lida com questões do seu mundo externo e interno (Burd, 2010; Conejo, 2009; Loli, 2000). Assim, a obesidade vista pelas lentes de uma concepção de saúde permeada por aspectos sociais, culturais e psicológicos pode ser considerada um recurso salutar e protetivo, não necessariamente definitivo, diante da vida e de conflitos psíquicos. 
Com esse olhar sobre a obesidade, considerar o êxito terapêutico (emagrecimento, alteração de IMC) como objetivo do cuidado é reduzir o adolescente a um corpo obeso e abandonar, dessa forma, uma concepção mais ampla de saúde. Nesse sentido, corrobora-se a afirmação de Ayres (2004, p. 24) de que "é preciso que cada profissional de saúde, . . . gestor ou formulador de política se interroguem acerca de por que, como e quanto se responsabilizam em relação aos projetos de felicidade daqueles cuja saúde cuidam. . ..". Faz-se então, urgente a superação da concepção reducionista e objetificadora da obesidade.

\section{Considerações Finais}

A compreensão de que a obesidade é, também, um sintoma psíquico influenciado por aspectos da dinâmica familiar e sociocultural esteve presente ao longo do trabalho. Foi possível compreender que o cuidado ofertado aos adolescentes com obesidade grave é realizado de forma fragmentada, denotando a compreensão de que a saúde mental e os aspectos sociais não são contemplados no âmbito da atenção básica. Encontraram-se percepções tanto de profissionais e familiares quanto dos próprios adolescentes que percebem o cuidado como efetivado, porém, entre o cuidado ofertado, que ainda é determinado pelo modelo biomédico, e o cuidado integral, que considera os aspectos psicossociais e as particularidades da adolescência, há um caminho a ser percorrido recheado de disputas teóricas e políticas.

Através de um olhar mais reflexivo, os resultados apontaram para dois horizontes. O primeiro denota a distância entre o discurso das políticas públicas e a organização de uma rede de cuidado integral. O segundo aspecto retratou a adolescência, etapa fundamental para o desenvolvimento do ser humano, como um período de singularidades que necessita de cuidados específicos. Este, porém, encontra-se à margem dessa prudência no que se refere à atenção básica.

Para que se efetive uma atenção básica que oferte cuidado integral e seja norteadora da rede de atenção, faz-se imperativa a presença de profissionais que possibilitem a ampliação da discussão sobre os processos de saúde- doença. Considerando a impossibilidade de esgotar o debate, mas vislumbrando mudanças futuras, entende-se que ampliar a oferta de cuidados em saúde mental e considerar os aspectos sociais na atenção básica possibilitará ganhos na qualidade da atenção integral à saúde direcionada ao adolescente com obesidade. O advento do SUS e do ECA inauguraram, para os adolescentes, o status de sujeito de direito, sendo necessário, agora, concretizar o seu status de sujeito integral.

\section{Referências}

Alves, I. F., \& Laguardia, N. (2010). Obesidade: Um expressar silencioso na clínica psicanalítica. Revista de Psicologia, 1(25).

Ayres, J. R. C. M. (2004). O cuidado, os modos de ser (do) humano e as práticas de saúde. Saúde e Sociedade, 13(3), 16-29.

Bardin, L. (2010). Análise de conteúdo (4a ed. rev. e atual.). Lisboa: Edições 70.

Brasil. Ministério da Saúde. (2006a). Secretaria de Atenção à Saúde. Departamento de Atenção Básica. Obesidade. Cadernos de atenção básica, 12.

Brasil, Ministério da Saúde (2010). Secretaria de Atenção em Saúde. Departamento de Ações Programáticas Estratégicas. Diretrizes nacionais para a atenção integral à saúde de 
adolescentes e jovens na promoção, proteção e recuperação da saúde. Série A. Normas e Manuais Técnicos. Brasília, DF.

Brasil, Ministério da Saúde. (2012). Secretaria de Atenção à Saúde. Departamento de Atenção Básica. Política Nacional de Alimentação e Nutrição. Série B, Textos Básicos de Saúde, Brasília, DF.

Brasil, Ministério da Saúde. (2013). Secretaria de Atenção à Saúde. Departamento de Atenção Básica Saúde mental. Cadernos de Atenção Básica, 34.

Brasil. Ministério da Saúde. (2014a). Organização Pan-Americana da Saúde. Perspectivas e desafios no cuidado às pessoas com obesidade no SUS: Resultados do Laboratório de Inovação no manejo da obesidade nas Redes de Atenção à Saúde. Serie Técnica Redes Integradas de Atenção à Saúde, 10.

Brasil, Ministério da Saúde. (2014b). Secretaria de Atenção à Saúde. Departamento de Atenção Básica. Guia alimentar para a população brasileira (2a ed.). Brasília, DF.

Brasil, Ministério da Saúde (2014c). Secretaria de Atenção à Saúde. Departamento de Atenção Básica. Estratégias para o cuidado da pessoa com doença crônica: Obesidade. Cadernos de Atenção Básica, 38.

Burd, M. (2010). Obesidade e família. In J. de Mello Filho, \& M. Burd (orgs.), Doença e família (2a ed., Cap. 17, pp. 299-310). São Paulo: Casa do Psicólogo.

Carneiro, H. S. (2005). Comida e sociedade: Significados sociais na história da alimentação. História: Questões \& Debates, 42(1), 71-80.

Conejo, S. P. (2009). Homem e obesidade-excesso e faltas: Corpos que contam histórias. (Tese de Doutorado, Universidade de São Paulo, São Paulo, SP).

Dornelles, A. D., Anton, M. C., \& Pizzinato, A. (2014). O papel da sociedade e da família na assistência ao sobrepeso e à obesidade infantil: Percepção de trabalhadores da saúde em diferentes níveis de atenção. Saúde e Sociedade, 23(4), 1275-1287.

França S. L. G., Sahade V., Nunes M., Adan, L. F. (2013). Adherence to nutritional therapy in obese adolescents: A review. Nutrición Hospitalaria, 28(4), 988-998.

Grejanin, D. K. M., Pezzo, T. H., Nastri, V., Sanches, V. P. P., Nascimento, D. D. G., \& Quevedo, M. P. (2007). As percepções sobre o "ser obeso" sob a ótica do paciente e dos profissionais da saúde. Revista Brasileira de Crescimento e Desenvolvimento Humano, 17(3), 37-47.

Kaufman, A. (2012, dez.). Alimento e emoção. Abeso, 60, 7-11. Disponível em http://www. abeso.org.br/pdf/revista60/alimento_emocao.pdf

Loli, M. S. A. (2000). A obesidade como sintoma: Uma leitura psicanalítica. São Paulo: Vetor. Mattos, R. (2012). Sobrevivendo ao estigma da gordura. São Paulo: Vetor.

Mendes, E. V. (2012). O cuidado das condições crônicas na Atenção Básica à Saúde: O imperativo da consolidação da Estratégia da Saúde da Família. Brasília: Organização Panamericana da Saúde- Representação Brasil.

Menin, M., Paschoarelli, L. C., \& Silva, J. C. P. (2011). Parâmetros antropométricos para o design de produtos destinados à acessibilidade de obesos. Revista Brasileira de Biometria, 29(4), 673-687.

Nunes, J., \& Pelizzoli, M. L. (2011). O fenômeno da saúde- O cuidado à luz da hermenêutica filosófica. In M. L. Pelizzoli (org.). Saúde em novo paradigma. Recife: EDUFPE.

Rodrigues, E. M., \& Boog, M. C. F. (2006). Problematização como estratégia de educação nutricional com adolescentes obesos. Cadernos de Saúde Pública, 22(5), 923-931. 
Schoen-Ferreira, T. H., Aznar-Farias, M., \& Silvares, E. F. M. (2010, jun.). Adolescência através dos séculos. Psicologia: Teoria e Pesquisa, 26(2), 227-234. Disponível em http://www. scielo.br/pdf/ptp/v26n2/a04v26n2.pdf

Segal, A., Cardeal, M. V., \& Cordas, T. A. (2002). Aspectos psicossociais e psiquiátricos da obesidade. Revista de Psiquiatria Clínica, 29(2), 81-89.

Simão, V. M., \& Mioto, R. C. T. (2016, mar.). O cuidado paliativo e domiciliar em países da América Latina. Saúde em Debate, 40(108), 156-169. Disponível em http://www.scielo.br/ scielo.php?pid=S0103-11042016000100156\&script=sci_abstract\&tIng=pt

Recebido: 18/07/2017

Última revisão: 07/02/2018

Aceite final: 09/02/2018

\section{Sobre as autoras:}

Adriana Lobo Müller - Mestre em Saúde Coletiva. Especialista em Psicologia e Saúde Mental Coletiva. Psicóloga. Professora substituta na Universidade Regional de Blumenau (FURB). E-mail: adrilmuller@hotmail.com, Orcid: http://orcid.org/0000-0002-9262-8422

Cláudia Regina Lima Duarte da Silva - Doutora em Enfermagem pela Universidade Federal de Santa Catarina (UFSC). Mestre em Educação pela Universidade Regional de Blumenau (FURB). Enfermeira. Professora titular da Universidade Regional de Blumenau (FURB), lecionando nos cursos de graduação e no Mestrado Profissional em Saúde Coletiva. E-mail: claudiaduarte1108@gmail.com, Orcid: http://orcid.org/0000-0002-4813-1603

Deisi Maria Vargas - Doutora em Medicina e Cirurgia (Pediatria) pela Universitat Autonòma de Barcelona (UAB). Médica. Professora titular na Universidade Regional de Blumenau (FURB), atuando nos cursos de graduação e no Mestrado Profissional em Saúde Coletiva. E-mail: deisifurb@gmail.com, Orcid: http://orcid.org/0000-0003-4389-2670 